\title{
Retraction Note: Differentiation analysis for estimating individual ancestry from the Tibetan Plateau by an archaic altitude adaptation EPAS1 haplotype among East Asian populations
}

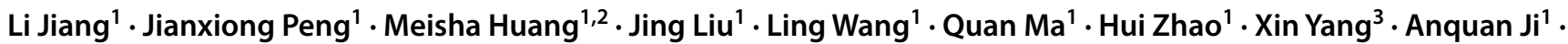 \\ Caixia $\mathrm{Li}^{1,2}$ (D)
}

Published online: 7 September 2021

(c) Springer-Verlag GmbH Germany, part of Springer Nature 2021

\section{Retraction Note to: International Journal of Legal Medi- cine (2018) 132:1527-1535 \\ https://doi.org/10.1007/s00414-018-1789-5}

The Editors-in-Chief have retracted this article. Since publication, concerns were raised about the ethics and consent procedures for this study. We requested supporting documentation from the authors, including the application form submitted to the ethics committee and evidence of ethics approval. The documents supplied by the authors contain insufficient information related to the scope of the study for us to remain confident that the protocols complied with our editorial policies or are in line with international ethical standards [1].

Caixia Li stated on behalf of all co-authors that they do not agree to this retraction.

\section{Reference}

1. World Medical Association. (2001). World Medical Association Declaration of Helsinki. Ethical principles for medical research

The original article can be found online at https://doi.org/10.1007/ s00414-018-1789-5.

\section{Caixia Li}

licaixia@tsinghua.org.cn

1 Key Laboratory of Forensic Genetics, Beijing Engineering Research, Center of Crime Scene Evidence Examination, National Engineering, Laboratory for Forensic Science, Institute of Forensic Science, Beijing 100038, People's Republic of China

2 Institute of Forensic Science, Shanxi Medical University, Taiyuan 036000, China

3 Lanzhou Public Security Bureau, Institute of Criminal Science and Technology, Lanzhou 730030, China involving human subjects. Bulletin of the World Health Organization, 79(4), 373.

Publisher's Note Springer Nature remains neutral with regard to jurisdictional claims in published maps and institutional affiliations. 